\title{
Clinicopathologic factors associated with short-term survival in advanced epithelial ovarian cancer
}

\author{
Shuwei Zhou ${ }^{1}$, Yao Liu ${ }^{2}$, Wanchun Yin $^{1}$, Qianqian Liao ${ }^{1}$, Quan Quan ${ }^{1}$, Xiaoling Mu ${ }^{1}$ \\ ${ }^{1}$ Department of Gynecology, The First Affiliated Hospital of Chongqing Medical University, Chongqing 400016, China; ${ }^{2}$ Department of Obstetrics \\ and Gynecology, Chengdu First People Hospital, Chengdu 610000, China \\ Contributions: (I) Conception and design: S Zhou, X Mu; (II) Administrative support: X Mu; (III) Provision of study materials or patients: Y Liu; \\ (IV) Collection and assembly of data: S Zhou, Q Liao, Q Quan; (V) Data analysis and interpretation: S Zhou, W Yin; (VI) Manuscript writing: All \\ authors; (VII) Final approval of manuscript: All authors. \\ Correspondence to: Dr. Xiaoling Mu. Department of Gynecology, The First Affiliated Hospital of Chongqing Medical University, No.1, YouYi Road, \\ Chongqing 400016, China. Email: muling99@163.com.
}

Background: Epithelial ovarian cancer (EOC) is the most lethal gynaecological malignancy, and there is a deficiency of information in the literature on the early recognition of short-term survivor (STS). This study aimed to identify the clinicopathological factors associated with STS in late-stage EOC and to establish a predictive model to identify STS.

Methods: Selected patients with International Federation of Gynecology and Obstetrics (FIGO) stage III or IV EOC were included in the study, and a retrospective analysis was performed. The characteristics of the patients who survived not more than 2 years (STS) were compared to those who survived at least 2 years (defined as long-term survivors, LTS). Binary logistic regression and receiver operating characteristic curve (ROC) were used to identify the independent prognostic factors associated with EOC and assess the predictive accuracy for STS.

Results: We identified 254 patients with advanced EOC including 57 STS and 197 LTS. A univariate analysis revealed that STS had a tendency to have omental metastasis and larger tumor size, to be platinum resistant, to have non-serous histology, to undergo suboptimal cytoreduction, to have comorbidity, and to undergo primary chemotherapy less than 6 courses. Binary regression analysis revealed that tumor size $(\mathrm{P}=0.033)$, platinum resistance $(\mathrm{P}<0.001)$, non-serous histology $(\mathrm{P}=0.048)$ and number of primary chemotherapy $(\mathrm{P}=0.028)$ were significant independent predictors of STS. A developed predictive model using these predictors had an $\mathrm{AUC}=0.831$; platinum resistance alone had an $\mathrm{AUC}=0.732$.

Conclusions: Tumor size, omental metastasis, platinum resistance, non-serous histology, and number of primary chemotherapy are predictors associated with STS when controlling other confounding factors. Tumor size and omental metastasis may be considered novel, important prognostic factors for advanced EOC patients. Platinum resistance was the most important prognosticator for STS; hence, more work is needed for the early identification and treatment of these EOC patients.

Keywords: Epithelial ovarian cancer (EOC); short-term survivor (STS); omental metastasis; tumor size; clinicopathological factors

Submitted Mar 10, 2019. Accepted for publication Sep 10, 2019.

doi: $10.21037 /$ tcr.2019.09.53

View this article at: http://dx.doi.org/10.21037/tcr.2019.09.53 


\section{Introduction}

A global broad scale study including 865,501 women from 61 countries recently reported that the 5 -year survival rate for ovarian cancer was between $30 \%$ and $50 \%$, and the survival trends were quite stable in most countries (1). Because epithelial ovarian cancer (EOC) has almost no early onset of symptoms and its current screening is difficult to be performed in the general population, nearly $80 \%$ of EOC patients were diagnosed during the late stage of the disease (2). After advanced EOC patients underwent cytoreductive surgery combined with platinum-based chemotherapy, about $15 \%$ of them could obtain 10 years survival after the diagnosis (3-5). In contrast, still 20-30\% of EOC patients who were diagnosed with the same disease and who underwent similar treatment had a survival rate not exceeding 2 years (6). Current clinical algorithms fail to distinguish these EOC patients from long-term survivors (LTS) at the time of diagnosis, and as a result, such patients and LTS are treated similarly. Hence, identifying the significant predictors of poor prognosis in EOC patients may lead to a development of more targeted therapeutic regimens and may improve the survival outcomes of EOC patients in the future.

Approximately 1,900 stage III aggressive EOC patients were included into a study conducted by Winter and colleagues; they identified the independent predictors of poor prognosis in stage III EOC patients, including mucinous or clear-cell histology, increasing age, impaired performance status, and gross residual disease (RD), which have been widely recognized so far (7). Additionally, recent extensive studies have focused on identifying the clinicopathological factors that LTS possessed in advanced EOC (4,8-11). While there is a deficiency of information in the literature on the early recognition of short-term survivor (STS) of advanced EOC to date, some prognostic factors of ovarian cancer were well-known, but we have hardly found any research on tumor size and omental metastasis that affected the overall survival (OS) in advanced EOC patients.

In this present study, we aimed to identify EOC patients who were considered as STS and the clinicopathological factors associated with their poor prognosis. Most prior studies were committed to exploring the clinical characteristics, tumor immune microenvironment, and germline variants of LTS (4,9-12), while ignoring the urgent healthcare need of STS. Identifying the significant predictors of STS with advanced EOC provides better personalized treatment. Physicians could perform direct trials to new treatments to EOC patients who are likely to be categorized as STS, supporting the ability of clinical trials to produce clinical benefits. This study aimed to identify the clinicopathological factors associated with STS and to improve the survival outcomes of EOC patients who are considered STS.

\section{Methods}

\section{Patient selection}

This retrospective study reviewed the medical records of women with International Federation of Gynecology and Obstetrics (FIGO, 2014) stage III or IV EOC who were treated at the First Affiliated Hospital of Chongqing Medical University, Chongqing, China, from March 1, 2011, to December 31, 2018. All of them underwent primary or interval cytoreductive surgery with or without adjuvant chemotherapy. We excluded women who merely underwent palliative chemotherapy, who had other malignant tumors, and who were followed up within 2 years after undergoing the primary treatment and were still alive. Patients with primary peritoneal cancer were also excluded. The study was approved by the Ethics Committee of the First Affiliated Hospital of Chongqing Medical University.

\section{Data collection}

STS refer to patients who survived not more than 2 years after initially undergoing cytoreductive surgery or first cycle of neoadjuvant chemotherapy. Patients who survived at least 2 years after undergoing the initial treatment were defined as LTS and were classified as the comparison group. We obtained patients' demographic and clinical information through the patients' electronic medical records from the hospital information system of the First Affiliated Hospital of Chongqing Medical University.

The database contains detailed demographic, clinical, and pathologic characteristics of the patients, including age at diagnosis, tumor size, histology, grade, body mass index (BMI), pretreatment serum CA-125 level, comorbidity, RD, ascites volume, platinum-free interval (PFI), chemotherapy administered, omental metastasis and treatment.

Tumors were graded as well (G1), moderately (G2), or poorly (G3) differentiated, but many clear-cell carcinomas and mucinous carcinomas were not graded in our medical records. Non-serous EOC included mucinous carcinoma, 
endometrioid tumor, clear-cell carcinoma, mixed, and carcinosarcoma. $\mathrm{RD} \leq 1 \mathrm{~cm}$ was found in optimal cytoreduction and RD $>1 \mathrm{~cm}$ in suboptimal cytoreduction. PFI was defined as the period from the cessation of primary platinum-based chemotherapy to recurrence or progression of the disease. PFI $<6$ months was defined as platinum resistant and $\mathrm{PFI} \geq 6$ months as platinum sensitive, respectively. OS refers to the time from the start of definitive diagnosis to death due to any cause. Omental metastasis was based on the pathologist's report using the postoperative specimens. Ascites volume was obtained from surgical record and was measured in milliliters. Comorbidity mainly refers to diseases of the heart, brain, liver, kidneys, and blood vessels, and the existence of one or more of the abovementioned diseases was defined as "yes", and the absence of any abovementioned diseases except for primary EOC was defined as "no". The BMI (weight in kilograms divided by the square of height in meters, $\mathrm{kg} / \mathrm{m}^{2}$ ) was calculated at diagnosis. Tumor size was obtained from the surgical records and calculated as the largest diameter of the primary lesion (centimeter); if the bilateral ovary or fallopian tube had a tumor, the sum of both sides was calculated.

\section{Statistical analyses}

Data were analyzed using SPSS version 25.0 (IBM, Armonk, NY, USA). Descriptive statistics were used to analyze the patients' demographic and clinical characteristics, and chisquared test or Fisher's exact test were used to analyze the categorical variables. Statistical significance of the continuous variables was assessed using the Wilcoxon signed-rank tests and was also summarized as the median with interquartile range (IQR). The independent prognostic factors of STS were determined using binary logistic regression models. The Cox proportional hazards model was used to estimate hazard ratios and $95 \%$ confidence intervals. Receiver operating characteristic (ROC) curves were used to assess the predictive accuracy of STS with advanced EOC. $\mathrm{P} \leq 0.050$ was considered to be statistically significant.

\section{Results}

\section{Patient characteristics}

A total of 430 patients with FIGO stage III or IV EOC and fallopian tube cancer were identified in the First Affiliated
Hospital of Chongqing Medical University from March 1, 2011, to December 31, 2018. We excluded 4 patients with other malignant tumors, 2 patients with breast cancer, 1 patient with leukemia, and 1 patient with lung cancer. A total of 3 patients who did not undergo primary surgery, 130 patients who were followed up for less than 2 years and still alive, and 39 patients who missed multiple variables were excluded. Finally, 57 STS (OS $\leq 2$ years) and 197 LTS (OS $>2$ years) were included in the analysis, and the median age of these patients was 52 years (Q1:47 to Q3:60 years). The median follow-up time was 35 months (Q1:26 to Q3:49 months), and a total of 187 (73.6\%) and 112 (44.1\%) patients, respectively, experienced disease recurrence and eventually died.

The clinicopathological characteristics of STS and LTS are described in Table 1. From this table, age at diagnosis, BMI, ascites volume, preoperative serum CA-125 level, neoadjuvant chemotherapy, and FIGO stage and tumor grade were similar between the two groups. Patients who were considered STS were more likely to have bigger tumor size and $\mathrm{RD}>1 \mathrm{~cm}$, to be platinum resistant, to have non-serous EOC, to undergo a number of primary chemotherapy less than six times, and to have omental metastasis and comorbidity. Majority of the patients underwent primary cytoreductive surgery, and all patients underwent platinum-based chemotherapy except for 2 STS, and 66 patients underwent neoadjuvant chemotherapy before surgery, and 21 of them were able to complete intermediate cytoreductive surgery. Most patients in the two groups presented with serous cancers, and 15 (26.3\%) non-serous subtypes were observed including 8 mucinous, 3 endometrioid, 2 clear cell, and 2 mixed in STS vs. 17 (8.6\%) non-serous subtypes in LTS $(\mathrm{P}<0.001)$. There were nearly seven times as many cases of stage III than those of stage IV, but grade information was missing in $18.1 \%$ of cases. Compared with LTS, the greater omentum was greatly invaded with cancer in STS, and only $5(8.8 \%)$ patients who were considered STS showed no omental lesions according to the pathology report. A total of $19(9.6 \%)$ LTS had a maximum tumor diameter of $\geq 20 \mathrm{~cm}$, and $13(22.8 \%)$ patients who were considered STS had also the same result $(\mathrm{P}=0.005)$. No obvious difference in the presence or absence of ascites volume was found between the two groups $(\mathrm{P}=0.878)$.

\section{Specific clinicopathological factors of STS}

Out of the 57 STS, 3 (5.2\%) of them died within 6 months 
Table 1 Comparison of clinicopathologic factors associated with short-term survival

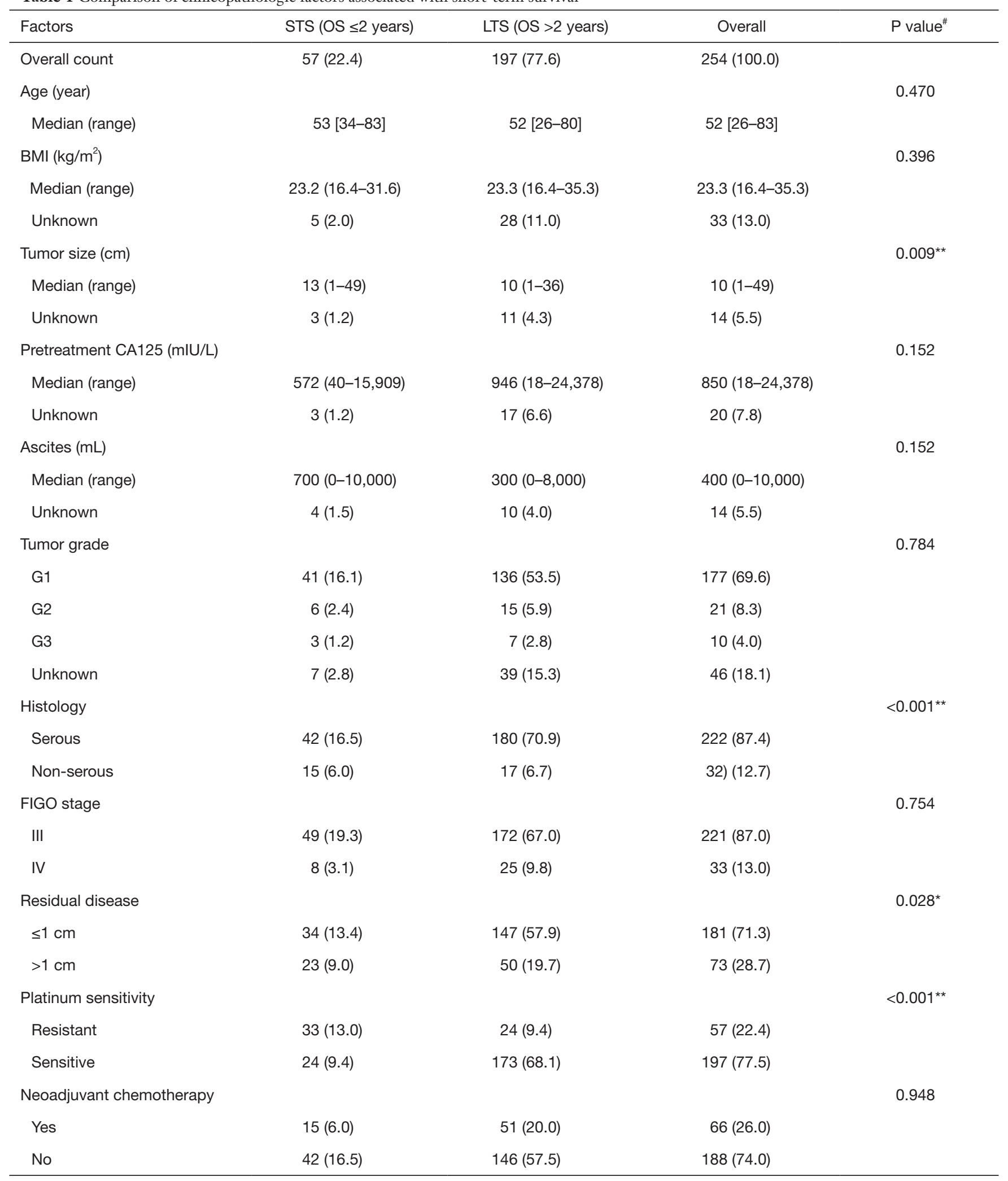

Table 1 (continued) 
Table 1 (continued)

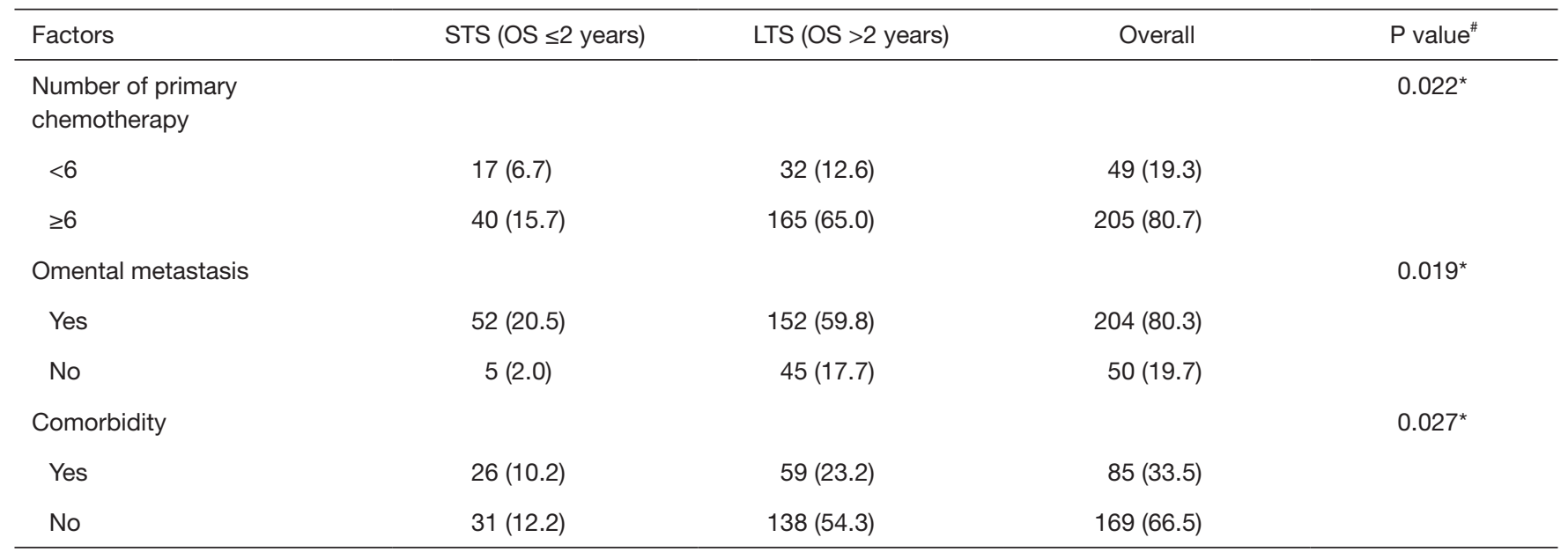

Data are resented as $\mathrm{n}(\%)$ or median (range). " , Wilcoxon signed-rank tests and chi-squared test were used to calculate the $\mathrm{P}$ values. *, $\mathrm{P}<0.05 ;{ }^{* *}, \mathrm{P}<0.01$. FIGO, International Federation of Gynecology and Obstetrics.

Table 2 Binary logistic regression models of prognostic factors

\begin{tabular}{lccc}
\hline Factors & OR $(95 \% \mathrm{Cl})$ & Comparison & Continuous \\
\hline Tumor size & $1.057(1.005-1.112)$ & $>1 \mathrm{~cm}$ reference: $\leq 1 \mathrm{~cm}$ & $0.033^{\star}$ \\
Residual disease & $1.090(0.481-2.470)$ & Sensitive ref: resistant \\
Platinum sensitivity & $0.083(0.036-0.191)$ & Serous ref: non-serous \\
Histology & $0.367(0.132-1.019)$ & $<6$ times ref: $\geq 6$ times \\
No. of CHT & $2.719(1.116-6.623)$ & Yes ref: no & $0.048^{\star}$ \\
Comorbidity & $1.629(0.739-3.587)$ & Yes ref: no & $0.028^{\star}$ \\
Omental metastasis & $3.214(0.931-11.103)$ & 0.065 \\
\hline
\end{tabular}

*, $\mathrm{P}<0.05$; ${ }^{*}, \mathrm{P}<0.01$. Covariate effects is the estimated odds ratio $(\mathrm{OR})$ for surviving not exceed 2 years. OR $>1$ indicates improved odds of STS relative to the comparison group. No. of $\mathrm{CHT}$, number of primary chemotherapy.

after the diagnosis; among them, one patient died of hypertension with cerebrovascular rupture, and the other 2 patients, who were 68 and 70 years old, had tumor sizes measuring 16 and $20 \mathrm{~cm}$, respectively; both of them had high-grade serous carcinomas, and optimal cytoreduction was the primary surgery, with omental metastasis caused by ovarian cancer progression, but one of them refused to undergo any chemotherapy. OS of $14(24.6 \%)$ STS was 6-12 months, the median age of these patients was 49 year, and tumor size in all these patients was $\geq 10 \mathrm{~cm}$ (median, $18 \mathrm{~cm}$ ); among these women, 13 (92.9\%) were confirmed by the pathologists to have omental metastasis, $10(71.4 \%)$ patients had RD $\leq 1 \mathrm{~cm}$, but 7 of them were platinum resistant; and the histological subtypes included the following: 7 serous, 4 mucinous, 2 mixed, and 1 endometrioid, that is to say, half of the mucinous STS (4/8) died within 1 year. In addition to the 17 patients mentioned above, the other 40 died between 12 and 24 months, and most of these women were platinum resistant.

\section{Binary regression analysis and predictive model of STS}

The final predictive model had seven main factors as shown in Table 2. Using a binary regression analysis, we found that an increase in tumor size was correlated with higher odds ratio of STS, independent of disease status. We also found that undergoing 6 courses or more of chemotherapy lowers the odds ratio (OR) of LTS and non-serous and platinum resistance 


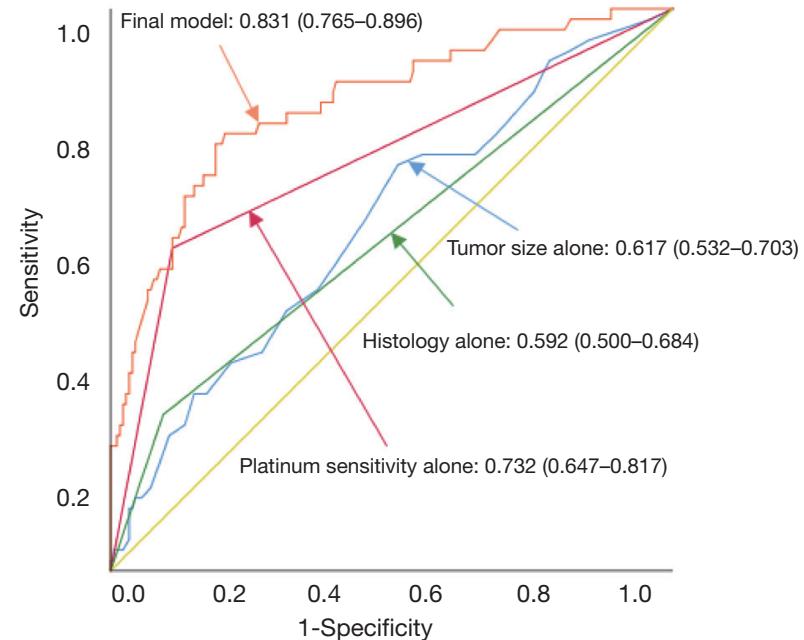

Figure 1 ROC curves of the final predictive model, compared to predictive ability of platinum sensitivity, tumor size and histology.

increases the OR of STS, independent of disease status. Our model did not demonstrate an independent association of RD, comorbidity, or omental metastasis with STS.

Predictive accuracy of the final model was assessed using the AUC. The final model using these independent variables had $\mathrm{AUC}=0.831$, as shown in Figure 1 . The other ROC curves in Figure 1 illustrate the influence of tumor size, platinum resistance, and histology on the predictive accuracy of the final model. Accuracy was strongly influenced by platinum resistance (alone AUC $=0.732$ ).

\section{Discussion}

Recently, substantial studies highlight the clinicopathological variables associated with long-term survival (some defined OS $\geq 5$ years, and others defined $O S \geq 10$ years) among advanced EOC patients (5,8-12), but there were few scholars who identified these characteristics of STS. In this study, we identified the demographic, surgical, pathologic, and treatment-related clinical factors associated with shortterm survival in advanced EOC. Our results revealed that patients who were considered STS were prone to have the following characteristics: platinum resistant, non-serous (especially mucinous type) histology, larger tumor size, suboptimal cytoreduction, omental metastasis, comorbidity, and primary chemotherapy less than 6 courses in univariate analyses. This finding is significant because it identified STS at the initial diagnosis and attempted to receive novel therapeutic regimen and improve OS.
Many prognostic factors for EOC are widely recognized, including FIGO stage, tumor grade, RD after initial surgery, age at diagnosis, and histologic types (7). Consistent with the majority of findings, patients who were considered STS underwent suboptimal cytoreduction, were platinum resistant, had comorbidity and non-serous histology, and did not finish the initial chemotherapy course (13-16). Population-based studies invariably owe long-term survival to optimal surgical cytoreduction and primary platinum resistance and, in some cases, aggressive surgical intervention after disease recurrence $(4,5,8-12)$. In terms of FIGO stage, tumor grade, and age, these were not associated with short-term survival in our analysis, possibly due to the effect of small sample size and short follow-up time. Recent data indicated that a large amount of ascites at the initial diagnosis of EOC was associated with worse prognosis, which may be related in part to decreased possibility of performing complete tumor resection as well (17). Additionally, ascites volume strongly promotes angiogenesis and increases cancer cells' transmesothelial invasion $(18,19)$, and a more interesting finding was that the expression of multidrug resistance associated protein in ovarian cancer cells was caused by increased ascites volume (20), but exact mechanism is yet unclear. Our results demonstrate the median of ascites volume in STS was $700 \mathrm{~mL}$ compared to that of LTS, which was $300 \mathrm{~mL}$, but there is no significant difference, and we need to include more patients to explore the impact of ascitic fluid volume on survival. A low pretreatment serum CA-125 level was a significant independent predictor in LTS (10); in contrast, STS had a slightly lower serum CA-125 level (median, 572 vs. $946 \mathrm{~m} / \mathrm{IU}$ ) in this present study, which maybe interfered by histological types. There is no consensus on the impact of obesity in EOC, and some studies confirmed that increasing BMI was associated with increased risk of death (21); nevertheless, Bandera et al. reported that obesity increased the survival among advanced EOC women (22).

Data are still dearth as regards the associations of tumor size with EOC survival, and a recent study reported that the normal-sized ovary was associated with poor OS compared with that of enlarged ovaries in EOC (23); earlier research illustrated that tumor volume had a predictive value for response to platinum-based chemotherapy and larger primary tumor volume was more likely to have a shorter time to progress in advanced EOC (24). Our current data revealed that STS have a tendency to have larger tumor size; in other words, tumor size may be a prognostic factor for EOC. 
The main site of ovarian cancer metastasis is the omentum, and abundant evidence suggests that the omentum functions as a niche that promotes ovarian cancer metastasis, and the main mechanism included increasing cell cycle duration and chemoresistance (25-27). Ferber reported that lymphangiogenesis in ovarian cancers appears to be affected by omental fat (28). To our knowledge, almost no one has studied the relationship between omental metastasis and OS so far in late-stage EOC, and this study revealed that STS had tendency a to undergo omental metastasis instead of other extrapelvic organ metastases. We will include more samples in different ways to explore whether omental metastasis is an independent prognostic factor in patients with advanced EOC.

When controlling for the other confounding factors, our study revealed that tumor size, platinum resistance, non-serous histology, number of primary chemotherapy, and omental metastasis were considered the significant predictors of STS in a multivariate analysis. The model's predictive (AUC, 0.83) model may discriminate STS adequately to change traditional treatments that might eventually improve clinical benefits (29). In sum, there are multiple avenues to become a STS, but the strongest factor may be primary platinum resistance (alone AUC, 0.73). Researchers like Slaughter and colleagues investigated 330 women with high-grade serous ovarian cancer and found that $25 \%$ of patients were primary platinum resistant (PPR), and the median PFS and OS of PPR patients were 4.2 and 17.8 months, respectively (30). Thomas et al. had similar report, PPR manifested earlier is a bad prognosis for EOC patients, and also found that neither age, primary treatment, $\mathrm{RD}$, and histology nor clinical trial was associated with PPR (31). Great progress has been made in recent years in making the mechanisms of platinum resistance clear in EOC, which mainly consist of constitutional germline mutations in BRCA1/BRCA2, epigenetic changes, and molecular anomalies of DNA repair pathways (32). Antiangiogenic therapies and poly (ADP-ribose) polymerase inhibitors were approved as new treatment options for ovarian cancer (33), and a randomized phase III trial called JAVELIN Ovarian 200 is being conducted to investigate the role of avelumab (anti-PD-L1) in platinum-resistant or refractory EOC patients (34). However, there are still no effective therapeutic approaches for patients with platinumresistant EOC, and it is necessary for us to eliminate any platinum resistant modifiable risk factors to improve patients' OS beyond the 24-month mark.

Limitations of our study include the following. First, this is a retrospective observational study, which may have potential recall and confusion bias. Second, the inclusion criteria for this study are not strict enough; hence, all patients should be treated similarly on a clinical trial to reduce some interference. Third, tumor size in surgical record may not be very accurate; thus, imaging examinations such as ultrasound or magnetic resonance imaging should be performed to make it more precise. Lastly, it was impossible to obtain the specimens of these STS to analyze the genetic factors such as the presence of BRCA mutations or other genomic data concerning chemosensitivity. Further exploration focusing on the gene and protein expression profiles of STS patients' tumors and integration of this knowledge with identifiable clinicopathological factors will hopefully point the way to improved OS for EOC patients.

In conclusion, the present study provided evidence to identify STS according to clinicopathological characteristics. Tumor size and omental metastasis may be novel, important prognostic factors for advanced EOC patients. Further studies are needed to explore genes or other markers that are correlative of platinum resistance. Additionally, more studies are needed to achieve reliable and feasible model into a clinically useful calculation.

\section{Acknowledgments}

Funding: The current study was supported by the Chongqing Science and Technology Commission (grant no. cstc2017shmsA130034).

\section{Footnote}

Conflicts of Interest: The authors have completed the ICMJE uniform disclosure form (available at http://dx.doi. org/10.21037/tcr.2019.09.53). The authors have no conflicts of interest to declare.

Ethical Statement: The authors are accountable for all aspects of the work in ensuring that questions related to the accuracy or integrity of any part of the work are appropriately investigated and resolved. The study was conducted in accordance with the Declaration of Helsinki (as revised in 2013). The study was approved by the Ethics Committee of the First Affiliated Hospital of Chongqing Medical University. Individual informed consent was waived due to the retrospective nature of the study.

Open Access Statement: This is an Open Access article 
distributed in accordance with the Creative Commons Attribution-NonCommercial-NoDerivs 4.0 International License (CC BY-NC-ND 4.0), which permits the noncommercial replication and distribution of the article with the strict proviso that no changes or edits are made and the original work is properly cited (including links to both the formal publication through the relevant DOI and the license). See: https://creativecommons.org/licenses/by-nc-nd/4.0/.

\section{References}

1. Allemani C, Matsuda T, Di Carlo V, et al. Global surveillance of trends in cancer survival 2000-14 (CONCORD-3): analysis of individual records for 37 513025 patients diagnosed with one of 18 cancers from 322 population-based registries in 71 countries. Lancet 2018;391:1023-75.

2. Torre LA, Trabert B, DeSantis CE, et al. Ovarian cancer statistics, 2018. CA Cancer J Clin 2018;68:284-96.

3. Cress RD, Chen YS, Morris CR, et al. Characteristics of Long-Term Survivors of Epithelial Ovarian Cancer. Obstet Gynecol 2015;126:491-7.

4. Javellana M, Hoppenot C, Lengyel E. The road to long-term survival: Surgical approach and longitudinal treatments of long-term survivors of advanced-stage serous ovarian cancer. Gynecol Oncol 2019;152:228-34.

5. Baldwin LA, Huang B, Miller RW, et al. Ten-year relative survival for epithelial ovarian cancer. Obstet Gynecol 2012;120:612-8.

6. Heintz AP, Odicino F, Maisonneuve P, et al. Carcinoma of the Ovary. Int J Gynaecol Obstet 2003;83:135-66.

7. Winter WE, Maxwell GL, Tian C, et al. Prognostic Factors for Stage III Epithelial Ovarian Cancer: A Gynecologic Oncology Group Study. J Clin Oncol 2007;25:3621-7.

8. Son JH, Kong TW, Paek J, et al. Clinical characteristics and prognostic inflection points among long-term survivors of advanced epithelial ovarian cancer. Int J Gynaecol Obstet 2017;139:352-7.

9. Dao F, Schlappe BA, Tseng J, et al. Characteristics of 10year survivors of high-grade serous ovarian carcinoma. Gynecol Oncol 2016;141:260-3.

10. Hamilton CA, Miller A, Casablanca Y, et al. Clinicopathologic characteristics associated with longterm survival in advanced epithelial ovarian cancer: an NRG Oncology/Gynecologic Oncology Group ancillary data study. Gynecol Oncol 2018;148:275-80.

11. Hoppenot C, Eckert MA, Tienda SM, et al. Who are the long-term survivors of high grade serous ovarian cancer? Gynecol Oncol 2018;148:204-12.

12. Yang SYC, Lheureux S, Karakasis K, et al. Landscape of genomic alterations in high-grade serous ovarian cancer from exceptional long- and short-term survivors. Genome Med 2018;10:81.

13. Zhou J, Wu SG, Wang J, et al. The Effect of Histological Subtypes on Outcomes of Stage IV Epithelial Ovarian Cancer. Front Oncol 2018;8:577.

14. Chang SJ, Bristow RE, Ryu HS. Impact of complete cytoreduction leaving no gross residual disease associated with radical cytoreductive surgical procedures on survival in advanced ovarian cancer. Ann Surg Oncol 2012;19:4059-67.

15. Melamed A, Manning-Geist B, Bregar AJ, et al. Associations between residual disease and survival in epithelial ovarian cancer by histologic type. Gynecol Oncol 2017;147:250-6.

16. Hosono S, Kajiyama H, Mizuno K, et al. Comparison between serous and non-serous ovarian cancer as a prognostic factor in advanced epithelial ovarian carcinoma after primary debulking surgery. Int J Clin Oncol 2011;16:524-32.

17. Szender JB, Emmons T, Belliotti S, et al. Impact of ascites volume on clinical outcomes in ovarian cancer: A cohort study. Gynecol Oncol 2017;146:491-7.

18. Mikuła-Pietrasik J, Uruski P, Szubert S, et al. The Proangiogenic Capabilities of Malignant Ascites Generated by Aggressive Ovarian Tumors. Biomed Res Int 2017;2017:2592496.

19. Mikuła-Pietrasik J, Uruski P, SzubertS, et al. Malignant ascites determine the transmesothelial invasion of ovarian cancer cells. Int J Biochem Cell Biol 2017;92:6-13.

20. Mo L, Pospichalova V, Huang Z, et al. Ascites Increases Expression/Function of Multidrug Resistance Proteins in Ovarian Cancer Cells. PloS One 2015;10:e0131579.

21. Previs RA, Kilgore J, Craven R, et al. Obesity is associated with worse overall survival in women with low-grade papillary serous epithelial ovarian cancer. Int J Gynecol Cancer 2014;24:670-5.

22. Bandera EV, Lee VS, Qin B, et al. Impact of body mass index on ovarian cancer survival varies by stage. $\mathrm{Br} \mathrm{J}$ Cancer 2017;117:282-9.

23. Paik ES, Kim JH, Kim TJ, et al. Prognostic significance of normal-sized ovary in advanced serous epithelial ovarian cancer. J Gynecol Oncol 2018;29:e13.

24. Andreopoulou E, Andreopoulos D, Adamidis K, et al. Tumor volumetry as predictive and prognostic factor 
in the management of ovarian cancer. Anticancer Res 2002;22:1903-8.

25. Cardenas C, Alvero AB, Pitruzzello M, et al. The omentum promotes ovarian cancer cell survival by increasing cell cycle duration and chemoresistance. Cancer Res 2017;77:1995.

26. Brewer MA, Sun G, Anderson M, et al. The omental microenvironment promotes ovarian cancer metastasis and enhances CD-133 mediated self-assembly of ovarian cancer spheroids. Cancer Res 2013;73:3953.

27. Nowicka A, Marini FC, Solley TN, et al. Human omentalderived adipose stem cells increase ovarian cancer proliferation, migration, and chemoresistance. PloS One 2013;8:e81859.

28. Ferber D, Lenoir B, Suarez-Carmona M, et al. Omental fat in ovarian cancer induces lymphangiogenesis. Eur J Cancer 2018; 92:S8.

29. Riester M, Wei W, Waldron L, et al. Risk prediction for late-stage ovarian cancer by meta-analysis of 1525 patient samples. J Natl Cancer Inst 2014;106:2504-11.

30. Slaughter K, Holman LL, Thomas EL, et al. Primary and acquired platinum-resistance among women with high grade serous ovarian cancer. Gynecol Oncol 2016;142:225-30.

31. Thomas ED, Slaughter KN, Gunderson CC, et al. Characterization of primary platinum resistance in an era of biologic agents and novel chemotherapeutic design. Gynecol Oncol 2014;133:91.

32. van Zyl B, Tang D and Bowden NA. Biomarkers of platinum resistance in ovarian cancer: what can we use to improve treatment. Endocr Relat cancer 2018;25:R303-18.

33. Cortez AJ, Tudrej P, Kujawa KA et al. Advances in ovarian cancer therapy. Cancer Chemother Pharmacol 2018;81:17-38.

34. Pujade-Lauraine E, Fujiwara K, Dychter SS, et al. Avelumab (anti-PD-L1) in platinum-resistant/refractory ovarian cancer: JAVELIN Ovarian 200 Phase III study design. Future Oncol 2018;14:2103-13.

Cite this article as: Zhou S, Liu Y, Yin W, Liao Q, Quan Q, $\mathrm{Mu} \mathrm{X}$. Clinicopathologic factors associated with short-term survival in advanced epithelial ovarian cancer. Transl Cancer Res 2019;8(6):2396-2404. doi: 10.21037/tcr.2019.09.53 